\title{
Eine akute Fazialisparese als mögliche Impfkomplikation bei einer Impfung gegen SARS-CoV-2
}

\section{Acute facial paresis as a possible complication of vaccination against SARS-CoV-2}

\author{
Autoren \\ Gerd Fabian Volk, Anna-Maria Kuttenreich, Maren Geitner, Orlando Guntinas-Lichius
}

Institut

Klinik und Poliklinik für Hals-Nasen-Ohrenheilkunde und Fazialis-Nerv-Zentrum, Universitätsklinikum Jena

online publiziert 11.05 .2021

Bibliografie

Laryngo-Rhino-Otol 2021; 100: 526-528

DOI 10.1055/a-1501-0470

ISSN 0935-8943

(c) 2021. Thieme. All rights reserved.

Georg Thieme Verlag KG, Rüdigerstraße 14,

70469 Stuttgart, Germany

Korrespondenzadresse

Univ.-Prof. Dr. med. Orlando Guntinas-Lichius

Klinik und Poliklinik für Hals-Nasen-Ohrenheilkunde

Universitätsklinikum Jena, Am Klinikum 1,

07747 Jena, Deutschland

Tel.: +49/3641/9329301

Fax: $+49 / 3641 / 9329302$

orlando.guntinas@med.uni-jena.de

\section{ZUSAMMENFASSUNG}

Obwohl die akute Fazialisparese (akute Gesichtsnervenlähmung, Bell-Lähmung) als mögliche Impfkomplikation auf eine Impfung gegen SARS-CoV-2 explizit im Aufklärungsmerkblatt der Impfstoffe erwähnt wird, überwiegt aus unserer Sicht klar der Nutzen der Impfung gegenüber möglichen Risiken. Aus den bisherigen Fallbeschreibungen lässt sich allenfalls, wenn überhaupt, ein gering erhöhtes Risiko ableiten. Das Risiko einer akuten Fazialisparese ist im Zusammenhang mit vielen Impfungen beschrieben; das Risiko scheint, wenn vorhanden, kein spezifisches Risiko zu sein. Andererseits sind auch Fälle von akuter Fazialisparese unter einer COVID-19-Erkrankung beschrieben, sodass sich die theoretische Frage stellt, inwieweit die Impfung möglicherweise eher das Auftreten einer Fazialisparese verhindert als befördert. Schlussendlich lässt sich die akute Fazialisparese, wenn schnell gehandelt wird, gut therapieren und ist von der Schwere und Folgeerscheinungen nicht mit der Krankheitsschwere und den möglichen Folgeerscheinungen einer COVID-19-Erkrankung zu vergleichen.

\section{ABSTRACT}

Although acute facial nerve palsy (Bell's palsy) is explicitly mentioned in the information sheets for vaccines as a possible complication of vaccination against SARS-CoV-2, from our point of view the benefits of the vaccination clearly outweigh the possible risks. At most, if at all, a slightly increased risk can be derived from the previous case reports. In general, the risk of acute facial palsy is described in association with many vaccinations. The risk, if any, does not appear to be a specific risk of SARS-CoV-2 vaccines. On the other hand, cases of acute facial palsy as symptom of a COVID-19 disease have also been described, so that the theoretical question arises as to the extent to which the vaccination may prevent rather than promote the occurrence of facial palsy. Ultimately, if acted quickly, acute facial paralysis can be treated well and its severity and sequelae cannot be compared with the severity of a COVID-19 disease and its possible long-COVID sequelae.

\section{Patienten wollen über das Risiko einer Fazialisparese aufgeklärt werden}

In den Medien, insbesondere in den sozialen Medien, werden von den Bürgern und Patienten sehr kontroverse Diskussionen über die Impfung gegen SARS-CoV-2 geführt. Es häufen sich Beschreibungen zum Auftreten einer akuten Fazialisparese nach der Impfung, und unsere Patienten fragen gehäuft nach dem Risiko. Daher sollten HNO-Ärztinnen und -Ärzte sorgfältig über das Thema aufklären können. Hier stellen wir die Fakten zusammen.

\section{Fallberichte zum Auftreten einer akuten Fazialisparese nach Impfung gegen SARS-CoV-2}

Es gibt Fallberichte, die das seltene Auftreten einer Fazialisparese in zeitlichem Zusammenhang mit der Gabe von mRNA-Vakzinen gegen SARS-CoV-2 beschreiben: In einer Phase-III-Studie mit dem BioNTech/Pfizer-Impfstoff wurden die Daten von knapp 38000 Probanden einbezogen, die über 2 Monate nach der zweiten Gabe (21 Tage nach der ersten Gabe) nachbeobachtet wur- 
den. In diesem Zeitraum gab es insgesamt 4 Fälle einer Fazialisparese in der Gruppe der Geimpften und keinen Fall in der PlaceboGruppe [1]. In der Zwischenauswertung einer weiteren Studie mit über 30350 Probanden zum Moderna-Impfstoff wurden 3 Fälle von Fazialisparese in der Impfgruppe dokumentiert und ein Fall in der Placebo-Gruppe [2]. Nachbeobachtet wurde durchschnittlich 7 Wochen nach der zweiten Gabe (durchschnittlich 29 Tage nach der ersten Gabe).

Im Detail betrachtet erlitten in der Studie von BioNTech/Pfizer von den insgesamt 18801 Personen, die den Impfstoff erhalten hatten, innerhalb des Nachbeobachtungszeitraums von ca. 82 Tagen 4 eine akute Fazialisparese. Die Fazialisparesen traten nach 3, 9, 37 und 48 Tagen nach der Impfung auf. In der Kontrollgruppe mit 18785 Personen wurde keine Fazialisparese dokumentiert.

In der Zwischenauswertung des Moderna-Impfstoffs erlitten von den insgesamt 15180 Personen, die den Impfstoff erhalten hatten, innerhalb des Nachbeobachtungszeitraums von ca. 78 Tagen 3 eine akute Fazialisparese. In der Kontrollgruppe mit insgesamt 15170 wurde im gleichen Zeitraum nur ein Fall von einer akuten Fazialisparese dokumentiert.

Beide Studienberichte deuten darauf hin, dass die Impfung im Vergleich zu einer Placebo-Gabe das Auftreten einer akuten Fazialisparese begünstigt. Werden beide Studien gemeinsam betrachtet, sind in den ca. 80 Tagen Nachbeobachtungszeit nach der ersten Impfung in der Impfgruppe 7 Fälle von akuter Fazialisparese aufgetreten, in der annähernd gleich großen Vergleichsgruppe nur ein Fall. Auch wenn bei diesen seltenen Ereignissen statistische Aussagen nur vorsichtig getroffen werden können, scheint das Risiko durch die Impfung um das 7-Fache erhöht zu sein [3]. Für den COVID-19-Impfstoff von AstraZeneca ist das Auftreten einer Fazialisparese bislang nicht berichtet worden. Für die anderen weltweit zugelassenen Impfstoffe haben wir keine Daten zum Auftreten einer Fazialisparese finden können.

\section{Vergleich der Inzidenz der akuten Fazialisparese mit der Inzidenz nach einer Impfung}

Das Risiko, eine akute idiopathische Fazialisparese zu erleiden, wird aber auch unabhängig von einer Impfung mit 7-40 Fällen pro Jahr auf 100000 Einwohner angegeben [4]. Es wurde daher bereits behauptet, die insgesamt 8 Fälle von akuten Fazialisparesen in der gesamten Studienpopulation von ca. 68000 Personen seien vergleichbar mit der spontanen Inzidenz von 7-40 Fällen pro 100000 Einwohnern pro Jahr [5]. Diese Überlegung berücksichtigt aber nicht, dass in den beiden Studien nur die Hälfte der Personen, d. h. 33982 Menschen, geimpft wurde und die Beobachtungszeit nur ca. 80 Tage statt 365 Tage betrug.

Werden die 7 berichteten Fälle von Fazialisparesen in der Impfgruppe mit 33982 Personen $(18801+15180)$ angenommen und auf 100000 Personen hochgerechnet, wären 20,6 Fälle von akuter Fazialisparese pro 100000 Impfungen zu erwarten. Rein statistisch sollten jedoch maximal 8,8 akute idiopathische Fazialisparesen in 80 Tagen Beobachtungszeit pro 100000 Einwohner auftreten, wenn die aufgetretenen Fälle (spontan versus nach Impfung) als vergleichbar betitelt werden. Das Risiko für eine akute Fazialisparese scheint also nicht nur im Vergleich zur Placebo-Gruppe, sondern auch in diesem Vergleich mit anderen Beobachtungsstudien zum zufälligen Auftreten von akuten idiopathischen Fazialisparesen vor COVID-19 um mindestens das 2,3-Fache erhöht zu sein (im Zeitraum 80 Tage nach den Impfungen).

\section{Es ist lange bekannt, dass eine akute Fazialisparese nach bestimmten Impfungen auftreten kann}

Wie dargelegt gibt es also deutliche Hinweise darauf, dass die SARS-CoV-2-Impfung das Risiko für eine akute Fazialisparese erhöht. Dies ist aber auch für andere Impfstoffe bekannt. Lähmungen des Gesichtsnervs wurden bereits als unerwünschtes Ereignis nach Impfungen gegen andere Erreger beschrieben, darunter Influenza, Hepatitis B, Polio, Diphtherie-Tetanus-Pertussis und azelluläre Pertussis sowie Masern-Mumps-Röteln [6].

\section{Möglicherweise ist das Risiko für das Auftreten einer akuten Fazialisparese durch eine SARS-CoV-2-Infektion erhöht}

Aber auch wenn eine Impfung eine Fazialisparese auslösen kann, muss bei der Nutzen-Risiko-Abwägung der Impfung beachtet werden, dass auch die Infektion mit SARS-CoV-2 selbst möglicherweise das Auftreten einer Fazialisparese begünstigen kann.

In einer italienischen Studie stiegen die Fälle von akuten Gesichtsnervenlähmungen im Zeitraum zwischen dem 27. Februar 2020 und dem 3. Mai 2020 in einer Region in Norditalien, die zu der Zeit ein Corona-Hotspot war, im Vergleich zum gleichen Zeitraum im Jahr 2019 um über 60 \% [7]. 2019 gab es 22 Fälle, 2020 dann 38 Fälle. In einer türkischen Studie konnten bei 24,3 \% von insgesamt 41 während der Pandemie erkrankten Patienten mit Fazialisparese Antikörper gegen SARS-CoV-2 im Blut nachgewiesen werden [8]. Somit wurde die Fazialisparese womöglich durch eine Coronavirus-Infektion ausgelöst. Bei allen Betroffenen war die Gesichtsnervenlähmung übrigens das einzige Symptom. Es wurden keine anderen COVID-19-typischen Symptome entwickelt.

Diese beiden Studien zu akuten Fazialisparesen bei SARS-CoV-2Infektionen ergeben nur ein unvollständiges Bild, lassen aber vermuten, dass das Risiko für eine akute Fazialisparese durch eine SARS-CoV-2-Infektion sogar höher ist als durch die Impfung. „Wie Herpes-simplex-, Gürtelrose- oder Erkältungsviren kann auch SARS-CoV-2 offensichtlich Fazialisparesen triggern“, schlussfolgerte Professor Dr. Peter Berlit, Generalsekretär der Deutschen Neurologischen Gesellschaft in einer Presseinformation zu Fazialisparesen infolge von Corona-Impfungen im April 2021 [5]. In dieser Stellungnahme wird auch davon ausgegangen, dass die Impfung ein möglicher, aber viel seltenerer Auslöser für das Auftreten von akuten Fazialisparesen sein kann. Es sei aber kein ursächlicher Zusammenhang nach der aktuellen Datenlage zu vermuten. Die akuten Fazialisparesen treten also wahrscheinlich als unspezifische Folge auf die 
Entzündungsreaktion nach der Impfung auf und nicht als direkte Folge auf den spezifischen Impfstoff [9].

Wenn jetzt noch berücksichtigt wird, dass die Impfungen zu über $90 \%$ vor den schweren, möglicherweise tödlichen Verläufen einer COVID-19-Erkrankung schützen, die Fazialisparesen sich dagegen in der Mehrzahl der Fälle unter der Gabe von Glukokortikoiden komplett zurückbilden, kann eine klare Impfempfehlung ausgesprochen werden [10].

\section{Impfung bei Patienten, die bereits eine Fazialisparese hatten}

Patienten, die bereits eine Fazialisparese hatten, haben rein statistisch ein leicht erhöhtes Risiko für eine erneute Fazialisparese [4]. Auch für diese Patienten mit rezidivierender oder chronischer Fazialisparese gilt die Impfempfehlung. Durch die Behandlungsmöglichkeiten sowohl der akuten Fazialisparese, aber auch der chronischen Formen, wenn es z. B. zu dauerhaften Schädigungen wie Synkinesien kommt, sollte die Fazialisparese als behandelbare Impfkomplikation bekannt und bewusst sein, aber nicht von einer Impfung abschrecken.

\section{Fazit für die Praxis}

- Möglicherweise ist das Risiko für das Auftreten einer akuten Fazialisparese nach einer SARS-CoV-2-Impfung mit einem der beiden zugelassenen mRNA-Impfstoffe leicht erhöht. Langzeitdaten nach mehr Impfungen sind abzuwarten.

- Viele andere Impfungen sind, wie lange bekannt, mit einem erhöhten Risiko für das Auftreten einer akuten Fazialisparese assoziiert. Wie immer ist der Nutzen der Impfung gegen dieses Risiko abzuwägen.

- Möglicherweise ist die akute Fazialisparese aber auch ein Symptom/eine Komplikation der SARS-CoV-2-Infektion selbst; dann würde die Impfung eher Fälle einer akuten Fazialisparese verhindern helfen.

- Tritt eine akute Fazialisparese nach einer Impfung auf, sollte die Behandlung dieser wie sonst auch leitliniengerecht mit Gluko- kortikoiden erfolgen, wenn keine Kontraindikation besteht. Die Prognose ist bei Therapiebeginn binnen 48-72 Stunden prinzipiell gut.

- Patienten, die in der Vergangenheit bereits eine Fazialisparese erlitten haben, sollten sich deshalb nicht von einer Impfung abhalten lassen und gegebenenfalls einen Vektorimpfstoff einem mRNA-Impfstoff vorziehen.

Interessenkonflikt

Die Autorinnen/Autoren geben an, dass kein Interessenkonflikt besteht.

Literatur

[1] https://www.fda.gov/media/144245/download

[2] https://www.fda.gov/media/144434/download

[3] https://www.thelancet.com/journals/laninf/article/PIIS14733099(21)00076-1/fulltext

[4] Heckmann JG, Urban PP, Pitz S et al. Idiopathische Fazialisparese (Bell's palsy). Diagnostik und Therapie. Dtsch Arztebl Int 2019; 116: 692-702. doi:10.3238/arztebl.2019.0692

[5] https://dgn.org/wp-content/uploads/2021/04/210413_PM_Fazialispare sen.pdf

[6] Rath B, Gidudu JF, Anyoti $\mathrm{H}$ et al. Facial nerve palsy including Bell's palsy: case definition and guidelines for collection, analysis, and presentation of immunisation safety data. Vaccine 2017; 35: 1972-1983

[7] Codeluppi L, Venturelli F, Rossi ] et al. Facial palsy during the COVID-19 pandemic. Brain Behav 2021; 11 (1): e01939 doi:10.1002/brb3.1939 Epub 2020 Nov 7. PMID: 33159420; PMCID: PMC7821573

[8] Islamoglu Y, Celik B, Kiris M. Facial paralysis as the only symptom of COVID-19: A prospective study. Am J Otolaryngol 2021; 42 (4): 102956 doi:10.1016/j.amjoto.2021.102956

[9] Shemer A, Pras E, Hecht I. Peripheral Facial Nerve Palsy Following BNT162b2 (COVID-19) Vaccination. Israel Medical Association Journal 2021: 143-144 im Internet: https://ima-files.s3.amazonaws.com/ 268773_455e40fd-d140-410f-b1e3-e640b3ee158d.pdf

[10] Lima MA, Silva MTT, Soares CN et al. Peripheral facial nerve palsy associated with COVID-19. J Neurovirol 2020; 26 (6): 941-944. doi:10.1007/ s13365-020-00912-6 